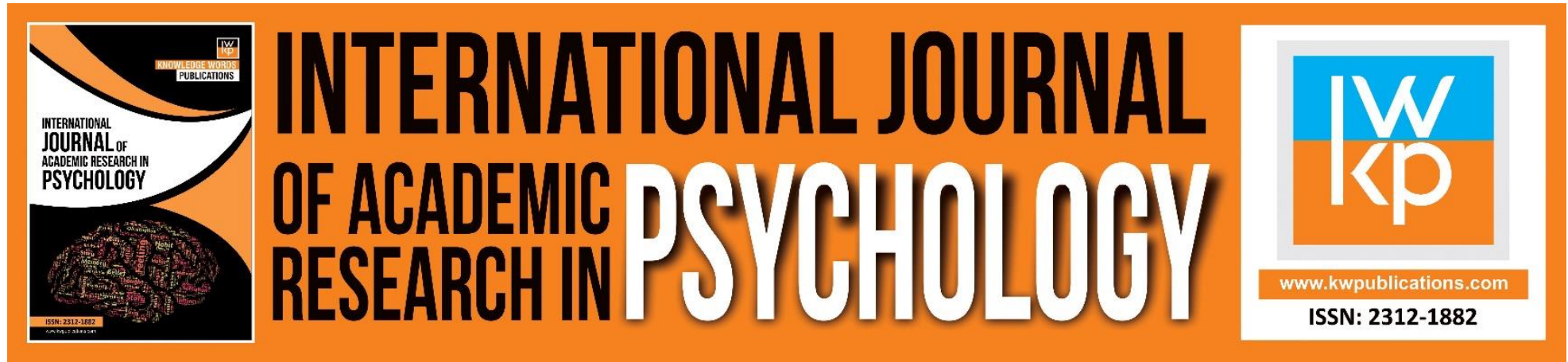

\title{
A Brief Review on Embodied Language Comprehension
}

\author{
Huili Wang, Yujiao Pan
}

To Link this Article: http://dx.doi.org/10.46886/IJARP/v4-i1/2095

DOI:10.46886/IJARP/v4-i1/2095

Received: 12 June 2017, Revised: 17 August 2017, Accepted: 30 August 2017

Published Online: 05 September 2017

In-Text Citation: (Wang, \& Pan, 2017)

To Cite this Article: Wang, H., \& Pan, Y. (2017). A Brief Review on Embodied Language Comprehension. International Journal of Academic Research in Psychology. 4(1), 30-42.

\section{Copyright: (C) 2017 The Author(s)}

Published by Knowledge Words Publications (www.kwpublications.com)

This article is published under the Creative Commons Attribution (CC BY 4.0) license. Anyone may reproduce, distribute, translate and create derivative works of this article (for both commercial and non-commercial purposes), subject to full attribution to the original publication and authors. The full terms of this license may be seen

at: http://creativecommons.org/licences/by/4.0/legalcode

Vol. 4, No. 1, 2017, Pg. 30 - 42

https://kwpublications.com/journals/journaldetail/IJARP

JOURNAL HOMEPAGE

Full Terms \& Conditions of access and use can be found at https://kwpublications.com/pages/detail/publication-ethics 


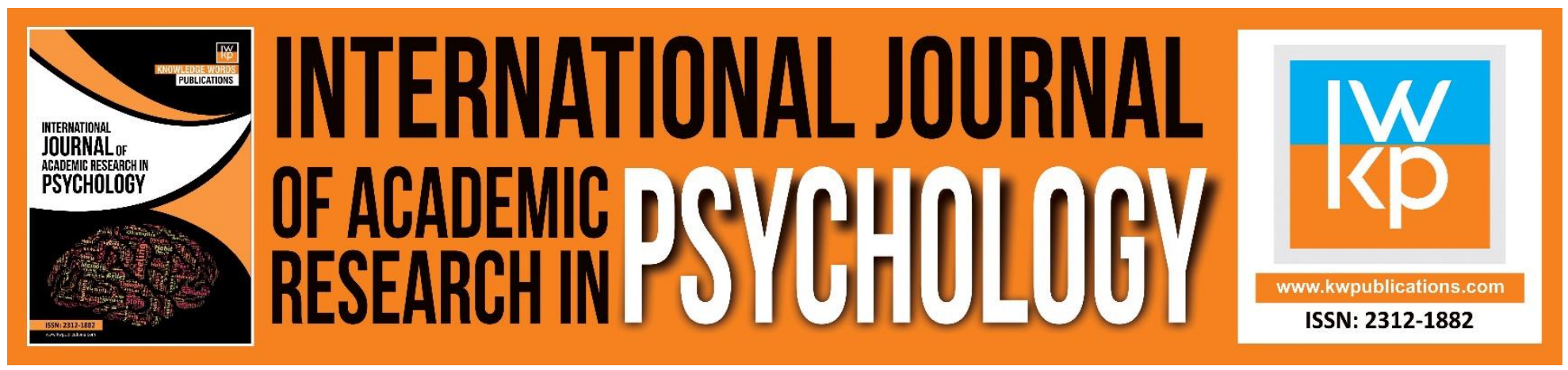

\title{
A Brief Review on Embodied Language Comprehension
}

\author{
Huili Wang, Yujiao Pan \\ Foreign Language School, Dalian University of Technology \\ Email: 1295097218@qq.com
}

\begin{abstract}
Recently, a growing body of research in psychology, psycho-linguistics and neuroscience has posed a challenge to the traditional view of language comprehension by proposing that cognitive states are not disembodied in language comprehension. Embodied theories of cognition hold that the actual mechanisms underlying language comprehension is hypothesized to entail performing mental simulations of its content. Numerous empirical researches have emerged in support of embodied view of language comprehension. While nowadays there is no single view of embodied cognition, its theories share many characteristics and assumptions (Wilson, 2002) and one of the most influential is Barsalou's (1999) Perceptual Symbol System, which proposes that people activate and manipulate perceptual symbols during language comprehension even when the perceptual characteristics are merely implied rather than explicitly stated. The purpose of this paper is to provide a systematic review of how sensory-motor and affective processes contribute to language comprehension.
\end{abstract}

Keywords: Embodied Theories, Language Comprehension, Perceptual Symbol System.

\section{Introduction}

Recently, a growing body of research in psychology, psycholinguistics and neuroscience has posed a challenge to the traditional view of language comprehension --- the Amodal Symbol Model, by proposing that cognitive states are not disembodied in language comprehension. Traditionally, language comprehension was supposed to be that linguistic inputs were mapped to semantic or conceptual representations. To understand an utterance, the language user maps words onto the semantic symbols that represent their meaning, and then are aligned as dictated by the sentence. Nonetheless advocates of current embodied cognition argue that language comprehension in essential reuses the conceptual and linguistic representations embedded in perceiving or acting, to be specific, it is a matter of reusing sensory-motor system. Accumulating behavioral and neuroscience evidence support the embodied view from research on language processing (Barsalou, 1999; Glenberg \& Kaschak, 2002; Kan et al., 2003; Gallese, 2008; Pecher \& Zwaan, 2005; Pulvermuller, 2008; Thompson-Schill, 2003). While nowadays there is no single view of embodied cognition, its theories share many characteristics and assumptions (Wilson, 2002) and one of the most influential is 
Barsalou's (1999) Perceptual Symbol System. Barsalou proposed that perceptual representations are the building blocks of cognition. Perceptual symbols or representations are the residues of a perceptual experience, stored in the brain and routinely activated in the course of language comprehension. Unlike amodal propositions, perceptual symbols bear an analog rather than arbitrary relationship with the referents. According to Perceptual Symbol System, the actual mechanisms underlying language comprehension is hypothesized to entail performing mental simulations of its content (Narayanan 1997; Barsalou 1999; Glenberg \& Robertson 2000; Bergen et al. 2004; Bergen \& Chang 2005). The purpose of this paper is to provide a systematic review of how sensory-motor and affective processes contribute to language comprehension.

\section{The Traditional View of Language Comprehension}

A classic debate in cognitive psychology and cognitive science has concerned how the information is stored and manipulated in the human brain. The historically prevalent theory of knowledge representation in cognitive science has been the amodal or (propositional) symbol system (e.g. Fodor, 1975; Kintsch, 1998; Newell \& Simon, 1972; Pylyshyn, 1981, 1984). Recently, however, researchers have posed a potentially viable alternative to amodal system in the form of perceptual symbol system (Barsalou, 1999), an embodied view of language comprehension. To clarify the embodied view of language comprehension, it is beneficial to contrast it with the traditional view in language comprehension.

As Gibbs (2006) puts it, the traditional belief of language comprehension is that meaning is an abstract entity divorced from bodily experience. Understanding language is assumed to require breaking down the physical information (e.g. speech sounds) into a language-independent medium that constitutes the "language of thought". The traditional view has been confirmed across different disciplines: in Al (Schank, 1972), in linguistics (Jackendoff, 1983, 1997, 2002), in psychology (Kintsch, 1998; Levelt, 1989; Miller \& Johnson-Laird, 1976), and in philosophy (Fodor, 1975; Latz, 1972; Katz \& Fodor, 1963). The most representative traditional view of language comprehension is the Amodal Symbol Model.

\section{Amodal Symbol Model}

The Amodal Symbol Model, emerging from the Cognitive Revolution in the 1950s remained dominant for over five decades in the area of language comprehension. The core assumption of the Amodal Symbol Model is that meanings of words and sentences are like a formal language, composed of abstract, amodal and arbitrary symbols, which stand for aspects of the word. (Burgess \& Lund, 1997; Chomsky, 1980; Fordor, 2000; Kintsch, 1998; Pinker, 1994). Take the word "banana" for example, word is abstract in that it refers to unripe green banana and ripe yellow banana, word is amodal in that the same word is used when banana is spoken about or written about, and word is arbitrarily related to the referents in that the phonemic and orthographic characteristics bear no relationship to the physical or functional characteristics of the word's referent. To understand it requires processing those amodal features of banana [fruit], [long], [curved], [yellow], [peel] etc., not retrieving the memory or experience of how it is typically perceived and used.

From this perspective, the mind is an abstract information processor and sensory-motor system are not related to high-level cognitive processes, like memory and language comprehension (Fodor, 1975; Newell \& Simon, 1976; Pylyshyn, 1984). Language comprehension was supposed to be that 
linguistic inputs were mapped to semantic or conceptual representations. To understand an utterance, the language user maps words onto the semantic symbols that represent their meaning, and then are aligned as dictated by the sentence. Semantic representations are fully symbolic and there is an arbitrary relationship between the word and the referent. The conceptual system, which is believed itself to be made up of such abstract, amodal symbols, is consequently updated on the basis of the new information that has just been entered into the system. The content of the utterance is understood and the semantic information is completely independent from sensory-motor system.

The Amodal Symbol Model was corroborated by several symbolic models describing how human memory is organized semantically and schematically (Bobrow \& Norman, 1975; Chariniak, 1978; Norman, 1975; Quillian, 1969; Rumelhart, 1975; Shank \& Abelson, 1995; Smith, Shoben \& Rips, 1974) as well as computational implementations, such as Knowledge Representation Language (Bobrow \& Winograd, 1977), Hyperspace Analog to Language (Lund \& Burgress, 1996), Topic Model (Griffiths \& Steyvers, 2004), and Latent Semantic Analysis (LSA) of Landauer and Dumais, 1997). Furthermore, the demonstrations of the most popular model, such as LSA, in picking out synonyms, measuring coherence of texts (Landauer and Dumais, 1997), and even scoring students' essay (Landauer, Laham, Rehder, \& Schreiner, 1997) led some scholars to support the potential of this model to account for human meaning (Landauer, 2002; Louwerse \& Ventura, 2005).

Nonetheless, the dominance of Amodal Symbol Model recently has been challenged by the embodied view of language comprehension, which proposes that language comprehension is grounded in mental representations of perceptual, motor and affective experiences of the world.

\section{The Embodied View of Language Comprehension}

Prior to the prevalent Amodal Symbol Model, however, ancient philosophers such as Epicurus (341-270 BC), on the contrary, proposed that concepts are rooted in modality-specific representations. This philosophical approach was resurrected in the 1990s and aroused scholars' interest ever since. It is the so called Perceptual Symbol System or Embodied Cognition or Simulation Model. The core proposal is that concepts are anchored in modality-specific system and language comprehension involves activating high-level perceptual and motor representations (Barsalou, 1999, 2008, Pecher \& Zwaan, 2005; Gibbs, 2006; Semin \& Smith, 2008; Shapiro, 2010).

Take the banana for example as well, understanding the object noun is supposed to involve activating modality-specific records in long term memory about how the banana looks, how it tastes, and how it is manipulated, etc. (Figure 1)

Figure 1. An illustration of Perceptual Symbol System, concepts are anchored in modality-specific system for perception and action (Based on a figure from Thompson-Schill et al., 2006, which itself was based on a figure from Allport, 1985.)

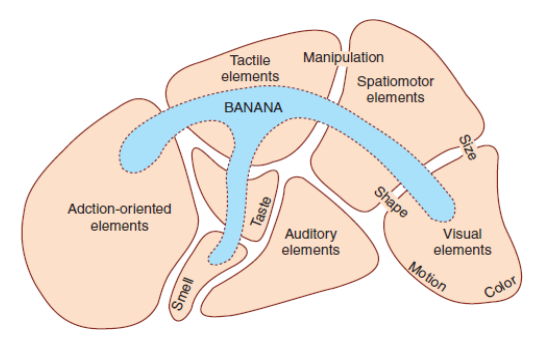

According to the Perceptual Symbol System, it is plausible that process a sentence, particularly a sentence involving an observable physical event, may often cause us to image the event being described.

There are three possible positions --- strong, moderate and weak in embodied vie of language comprehension that deal with the 
INTERNATIONAL JOURNAL OF ACADEMIC RESEARCH IN PSYCHOLOGY

Vol. 4, No. 1, 2017, E-ISSN: 2312-1882 @ 2017 KWP

relationship between enactive simulation and linguistic understanding. They are:

Strong embodied view of language comprehension (ELCS): Linguistic understanding just is an enactive simulation process;

Moderate embodied view of language comprehension (ELCm): linguistic understanding requires, but is not identified with, enactive simulation;

Weak embodied view of language comprehension (ELCW): linguistic understanding can use, but does not require, enactive simulation.

\section{Perceptual Symbol System}

In the past decade, numerous research in psychology, neuroscience and cognitive linguistics has grown exponentially, providing profound evidence for the embodied view of language processing (Barsalou, 1999; Boulenger et al., 2008; Borghi, 2004; Bub and Masson, 2010; de Vaga, 2008; Glenberg \& Kaschak, 2002; Pecher \& Zwaan, 2005; Pecher, Zeelenberg and Barsalou, 2003; Zwaan and Tayor, 2006). While nowadays there is no single view of embodied cognition, and one of the most influential is Barsalou's (1999) Perceptual Symbol Systems.

Barsalou argued that perceptual representations rather than the amodal propositions are the building blocks of cognition. Perceptual symbols are the residues of a perceptual experience, stored as patterns of activation in the brain. Because our attention is limited, perceptual symbols are typically schematic, rather than being like high-resolution video clips or high-fidelity sound clips. However, unlike amodal propositions, perceptual symbols bear an analog relationship with the referents. Barsalou assumed that perceptual symbols are used in perceptual simulations that make up human cognitive processes

In the cognitive psychology and cognitive linguistics domain, this activation of brain systems dedicated to perception or action has been interpreted as reflecting mental simulation of the content of the sentence on the part of the individual (Glenberg \& Kaschak, 2002; Zwaan, Stanfield \& Yaxley, 2002; Richardson et al., 2003; Bergen et al., 2007, Mahon \& Caramazza, 2008). Mental simulation is the internal enactment or reenactment of perceptual, motor or affective experiences (Barsalou, 1999). Mental simulation may be static or dynamic, and this term also called mental imagery in the literature. In the neuroscience, mental simulation is produced by brain structures specific to the relevant modality; motor simulation uses motor areas, down to the specific regions that control simulated effectors. Similarly, visual simulation is produced through activation of visual areas. It is noteworthy that while the perceptual and motor content of mental simulations can often be made accessible to conscious introspection, mental simulation constructed during language comprehension is immediate, automatic, and entirely unconscious (Barsalou, 1999). Perceptual Symbol System proposes that our knowledge about the world is developed not in a holistic but a categorical way because our attentions system focuses on components of experience in the context of possible interactions with the world. The continuous experience with the world, in turn, leads to gradual integration of perceptual symbols into a distributed multi-modal system that represents the category as a whole - a simulator (Barsalou, 2009). Thus we develop various kinds of perceptual simulator (visual, motor, emotional, etc.) and later get integrated with simulator for the words they refer to. Under this account, it is quite easy to distinguish between the color of a bear in the wood and the color of a bear in the polar and to discriminate between a sound of voice in a cave and a sound of voice in a room. In sum, it is the interaction between language, body and environment that 
INTERNATIONAL JOURNAL OF ACADEMIC RESEARCH IN PSYCHOLOGY

Vol. 4, No. 1, 2017, E-ISSN: 2312-1882 @ 2017 KWP

makes it possible for humans to make inferences about information implied by the sentence.

\section{The Behavioral Research on Perceptual Symbol System}

There is ample evidence on perceptual symbol system that people routinely perform perceptual simulation implied by the sentence during language comprehension. There are three major compatibility effect providing support for the embodied view of language comprehension: appearance, affordance, and action compatibility effect (the ACEs). Appearance compatibility effect is the methodology mainly adopted in the studies on perceptual symbol system. The rationale is a sentence-picture mapping: after reading or listening a sentence, an image of the object that either integratible or not integratible with the sentence presented, the task for the participants is to judge whether the object was mentioned in the proceeding sentence.

In a study by Standfield \& Zwaan (2001), they asked participants to read a sentence implying the orientation of an object either vertical or horizontal (e.g. "Mary hammered the nail into the floor" or "Mary hammered the nail into the wall"), each followed an image of object either match or mismatch the the orientation implied by the sentence, then participants judge whether the object was mentioned in the proceeding sentences by pressing a key labeled "Yes" or "No" on the computer. The findings are that there is a significant differences on the response latency between in the matched condition and in the mismatched condition. Participants were faster to respond to the image stimuli when matched the orientation implied by the sentence rather than mismatched. The findings suggest that people mentally represent the orientation of an object implied by the sentence.

A similar appearance compatibility effect was found in related studies of Zwaan, Standfield, and Yaxley (2002) on the simulation of object's shape. Participants read sentence describing an object with different shape (e.g. "The ranger saw the eagle in the sky" or "The ranger saw the eagle in the nest"). Then they saw an image matched or mismatched the shape of the object implied by the sentence. When the image matched the shape in the described scenario, participants are faster to respond.

The appearance compatibility effect also confirmed with the dynamic stimuli of object motion. Zwaan, Madden, Yaxley and Aveyard (2004) instructed participants to read sentences implying an object's motion towards or away from them (e.g. "You throw the ball to John" or "John throws the ball to you"), then presented two slides of an object in a sequence that makes it appear as if the object is coming closer or moving farther away. Participants are faster to that the same object in each slide when the direction of motion depicted agrees with the direction that the object would be moving in the scenario described by the sentence. A similar Match advantage was also found by Yaxley and Zwaan (2007) in related study on the simulation of visibility (e.g. "Through the fogged goggles, the skier could hardly identify the moose" or "Through the clean goggles, the skier could hardly identify the moose" )

Finally, in the most recent studies Zwaan and Pecher (2012) replicated and Engelen, Bouwmeester, de Bruin, and Zwaan (2011) extended the study of Standfield and Zwaan (2001); Zwaan, Standfield, and Yaxley (2002). Despite the research conducted by Louise Connell (2007) addressed a different claim to previous studies on perceptual symbol system that there was a mismatch advantage during language comprehension. He argued that color, the secondary object property is represented distinct from primary object properties, like shape, orientation and motion. He suggested that perceptual color perceptual information is activated during language 
INTERNATIONAL JOURNAL OF ACADEMIC RESEARCH IN PSYCHOLOGY

Vol. 4, No. 1, 2017, E-ISSN: 2312-1882 @ 2017 KWP

comprehension even doing so does not facilitate task performance: participants respond more quickly when the color of a pictured object mismatched the color implied by the previous sentence. Obviously all these behavioral evidence provides a support for the assumption of Perceptual Symbol System that language comprehension involves constructing sensory-motor simulations of a described scenario.

\section{The Neuroscientific Research on Perceptual Symbol System}

In spite of the behavioral evidence on Perceptual Symbol System, neuroscientific research provides substantial support for the idea that the same sensory-motor regions of the brain get activated during language comprehension (Eskenazi et al., 2009; Gallese, 2008; Kan et. al., 2003; Martin, 2001, 2007; Pulvermuller, 2008; Thompson-Schill, 2003). Since the early 1990s, an increasing number of research has applied various brain mapping techniques to investigate predictions about the degree to which language processing reuses modality-specific systems for perception and action. In what follows, we consider major findings of these research, with special reference to several kinds of semantic features which enter into the meaning of concrete nouns. Consider the Perceptual Symbol System, what we studied in present study, we mainly review three types of visual features -- color, shape and motion.

\section{Color Features}

Many kinds of objects have "typical" and "canonical " colors. It goes for numerous artifacts, whose colors are determined by social conventions (e.g., yellow taxis) and even more strongly to diverse categories of animals (e.g., white geese) and plant (e.g., red strawberries). Such object-color associations plays a critical part of people's semantic knowledge of the relevant nouns.

With regard to the brain, although color perception is mediated by many neural mechanisms which begin in the retina, two main cortical regions are particularly significant. First, passive color sensation, which takes place when a person simply gazed at flowers in the garden, depends on area V4, a patch of cortex residues in the lingual gyrus of the occipital lobe. Functional neuroimaging research has shown that this area is engaged more when people perceive colored stimuli than when they perceive grayscale equivalents (Zeki et al., 1991; Kleinschmidt et al., 1996; Hadjikhani et al., 1998). Moreover, neuropsychological research has indicated that damage to area V4 leads to achromatopsia, which is an impairment of the capacity to consciously view color (Zeki, 1990; Bouvier \& Engel, 2006). Second, active color sensation, which takes place when a person attentively, deliberately compares the shades of different colors, relies on the area V4, the middle sector of the fusiform gyrus, which is a part of the ventral temporal cortex. The fusiform gyrus constitutes much of the so called "what" pathway, that is the branch of visual processing hierarchy that deals with shape, color and texture properties of objects. The area V4 in the region of fusiform gyrus become active during color discrimination.

Whether the two main color perception areas --- V4 and V4a --- engaged when a person retrieves semantic knowledge about the color features of object encoded by nouns like taxis, geese, and strawberries? To solve this question, Simmons et al. (2007) conducted an fMRI study. The fMRI data showed that these dual criteria were satisfied by a large cluster of voxels in the left mid-fusiform gyrus, most likely overlapping V4a, which conforms to the Perceptual Symbol System, since it suggests that semantic knowledge is anchored in the brain's modality-specific systems. Nonetheless, 
opponents of the Perceptual Symbol System argued that fusiform activity observed in the color property judgments may not reflect the unconscious, implicit retrieval of conceptual color features per se, instead reflect the conscious, explicit generation of color imagery, a process that may occur after the relevant color knowledge has been accessed from a purely abstract semantic system located elsewhere in the brain. To further address the question, Simmons et al (2007) invoke some relevant neuropychological data. They found that damage to the left fusiform can lead to color agnosia, a disorder that impairs knowledge of the sorts of canonical object-color associations. This finding certainly bolsters previous fMRI study that the fusiform activity reflects the retrieval of conceptual color features, as opposed to only color imagery.

\section{Shape Features}

Overall, the most critical visual-semantic component of object nouns is shape (Vinson \& Vigliocco, 2008; Gainotti et al., 2009, 2013; Hoffman \& Ralph, 2013). Numerous research with diverse brain techniques proved that like color properties, the shape properties of visual objects are also represented in the ventral occipital temporal cortex. As the researchers put it, distinct regions of the fusiform gyrus were activated not only by images but also by words, which fit the prediction of the Perceptual Symbol System.

The convergent results of the research by Chao et al. (1999) and Wheatley et al. (2005) confirmed that the shape features of the meaning of the object nouns are captured by neurons in the ventral temporal cortex not only overlapping partially with those facilitates visual perception of the very same features, but also segregating according to semantic category. Further evidence in favor of the idea comes from fMRI research (Kan et al., 2003; Devlin et al., 2005; Mechelli et al., 2006; Noppeney et al., 2006; Mahon et al., 2007; Chouinard \& Goodale, 2010; Peelen et al., 2013; Tyler et al., 2013). Moreover, neuropsychological research has conformed to the Perceptual Symbol System that damage to the mid-fusiform gyrus, especially in the left hemisphere, frequently impairs the understanding of concrete object nouns (Gainotti, 2006; Capitani et al., 2009).

\section{Motion Features}

Another important visual-semantic component of object nouns is the characteristic motion patterns of the designated entities. For example, part of the meaning of rabbit is the typical hopping movement of this kind of animal, and part of the meaning of scissors is the idiosyncratic cutting movement of this kind of tool. Numerous research has conformed that area $\mathrm{MT}_{+}$located in the vicinity of the anterior occipital and lateral occipital sulci is activated in the passive perception of moving visual stimuli. Damage to this area can lead to akinetopsia, that is acquired motion blindness, an impaired ability to consciously see motion. There are two parallel motion processing pathways: pSTS (the pathway extends from $\mathrm{MT}_{+}$into a sector of the posterior superior temporal sulcus) responds to the sight of biological (e.g. animal) motion patterns and pMTG (the pathway extends from MT+ into a sector of the posterior middle temporal gyrus) responds to the sight of nonbiological (e.g. tool) motion patterns (Beauchamp \& Martin, 2007, Saygin, 2012). Consistent with the prediction of Grounder Cognition Model, evidence from the fMRI study suggested that two motion processing pathway, pSTS and pMTG contribute not only to the high-level perception, but also to the long-term semantic representation of category-specific object-motion associated patterns.

To sum up, the studies reviewed above well supports the prediction of the Perceptual Symbol 
System that the meanings of object nouns are anchored in modality-specific brain systems, thereby language comprehension involves activating perceptual and motor representations that. According to Perceptual Symbol System, object concepts are not representations stored in an autonomous semantic module, whereas they consist of multiple fragments of information, which are widely distributed across the cerebral cortex in a manner dictated by the content. Thus color features stored in the same part of ventral temporal cortex that underlie high-level color perception; shape features stored in the same part of ventral temporal cortex that underlie high-level shape perception; motion features stored in the same part of lateral temporal cortex that underlie high-level motion perception. This interpretation of conceptual knowledge suggests that when processing an object noun with complex multimodal features, for example, an animal word like "bear", the corresponding complex network of multimodal cortical area is rapidly and unconsciously engaged. Indeed, the Perceptual Symbol System proposes that it is precisely the activation of perceptual and motor representations that constitutes a critical part in language comprehension.

\section{Another two Prevalent Models in Strong Embodied Language Comprehension}

Perceptual Symbol System, a strong embodied view of language comprehension, which suggests that human cognition is completely grounded in sensory-motor systems and language comprehension involves activating high-level perceptual and motor representations. To our knowledge, alongside Perceptual Symbol Theory of Barsalou (1999), there are another three strong embodied theories currently arouse hot debates on language processing, namely Indexical Hypothesis of Glenberg and Robertson $(1999,2000)$ and Immersed Experienced Framework of Zwaan (2004).

Indexical Hypothesis (IH) of Glenberg and Robertson $(1999,2000)$ is another theory of strong embodied view of language comprehension that further develops the Perceptual Symbol System of Barsalou (1999). IH focuses on specifying the perceptual symbols related to action, especially the function of action in language comprehension. It was motivated by Glenberg's (1997) assumption that a situation becomes meaningful relying on the set of actions available to a particular person in a particular situation.

(IH): The first step in language comprehension is to index words and phrases to objects, analogical representations of the objects such as images, or to perceptual symbols (Barsalou, 1999; Stanfield \& Zwaan, 2001). Unlike abstract symbols, perceptual symbols are modal and non-arbitrary based on the perception of the referents. The second step is to derive affordances from the perceptual symbols (Glenberg \& Robertson, 2000; Kaschak \& Glenberg, 2000). Unlike the arbitrary symbols, new affordance can derive from the perceptual symbols because perceptual symbols are not arbitrarily related to the referents. The third step is to mesh those affordances under the guidance of syntactic constructions (Kaschak \& Glenberg, 2000).

Immersed experienced framework (IEF) of Zwaan is another theory of language comprehension in Embodied Cognition. It is a comprehensive theory of how embodied processes might work during language comprehension.

(IEF): Language is a set of cues to the comprehender to construct an experiential (perception plus action) simulation of the described situation. In this conceptualization, the comprehender is an immersed experiencer of the described situation, and comprehension is the vicarious experience of the described situation. The IEF puts forward that there are three major processes during language 
comprehension: activation, construal, and integration. During activation, target words activate the functional webs, the various original experience with the referent in different visuospatial configurations such as shape, orientation, color, motion, etc. During construal functional webs are integrated in simulation of the event implied by the language. Finally, integration refers to experientially-based transitions from one construal to another. And successful integrations are influenced by personal experience, amount of overlap (refers to how much of current mental simulation has the same components of construal as the previous simulation), predictability (anticipation of next event), and linguistics cues (tense, word order, grammatical markers, etc.)

\section{Conclusion}

To sum up, by contrasting the traditional and embodied view of language comprehension, we conclude that embodied language comprehension assumes an analogue relationship between a symbol and its referent, whereas Amodal Symbol System assumes an arbitrary relationship between a symbol and its referent. According to Perceptual Symbol System the complete representation of an object, called mental simulation, should reflect physical characteristics of the object. Amodal Symbol System, in contrast do not make this prediction. And in the past decade accumulating research in cognitive psychology and neuroscience have confirmed the embodied view of language comprehension, especially the Perceptual Symbol System from the perspective of object properties such as shape, orientation and motion. Actually there is substantial support from both behavioral and brain imaging research in favor of the notion that language comprehension is on the basis of unconscious and automatic internal recreation of previous, embodied experiences, using brain structures dedicated to perception and action. Undoubtedly there will be hot debates about the importance of symbolic representation and embodied representations in language comprehension. However, in the light of the previous research, it is possible that we overestimate the role of embodied factor or enactive simulation in language comprehension. Regarding to this issue, the research in the domain of embodied cognition will focus on providing compelling evidence for the role of simulation in deeper language comprehension in the future. And recently computational modeling has achieved research on this topics, which could provide a firmer theoretical support for the embodied approach to language comprehension.

\section{References}

Barsalou, L. W. (1999). Perceptual symbol system. Behavioral \& Brain Sciences, 22, 577-660.

Barsalou, L. W., Simmons, W. K., Barbey, A., \& Wilson, C. D. (2003). Grounding conceptual knowledge in modality-specific systems. Trends in Cognitive Sciences, 7, 84-91.

Barsalou, L. W., \& Wiemer-Hastings, K. (2005). Situating abstract concepts. In D. Pecher \& R. A. Zwaan (Eds.), Grounding cognition: The role of perception and action in memory, language, and thinking (pp. 129-163). Cambridge: Cambridge University Press.

Beauchamp, M. S., \& Martin, A. (2007). Grounding object concepts in perception and action. Cortex, 43, 461-468.

Bergen, B., Chang, N., \& Narayan, S. (2004). Simulated Action in an Embodied Construction Grammar. Proceedings of the Twenty-Sixth Annual Conference of the Cognitive Science Society.

Bergen, B., \& Chang, N. (2005). Embodied Construction Grammar in Simulation -Based Language Understanding. In J.-O. Ostman \& M. Fried (Eds.), Construction Grammars: Cognitive 
INTERNATIONAL JOURNAL OF ACADEMIC RESEARCH IN PSYCHOLOGY

Vol. 4, No. 1, 2017, E-ISSN: 2312-1882 @ 2017 KWP

grounding and theoretical extensions. John Benjamins.

Biederman, I., and Ju, G. (1988). Surface versus edge-based determinants of visual recognition. Cognit. Psychol. 20, 38-64

Borhi, A. M., Glenberg, A. M., \& Kaschak, M. P. (2004). Putting words in perspective. Memory and Cognition, 32, 863-873.

Bouvier, S. E., \& Engel, S. A. (2006). Behavioral deficits and cortical damage loci in cerebral achromatopsia. Cerebral Cortex, 16, 183-191.

Burgess, C., \& Lund, K. (1997). Modeling parsing constraints with high-dimensional context space. Language and Cognitive Processes, 12, 177-210.

Capitani, E. (2009). Posterior cerebral artery infarcts and semantic category dissociations: A study of 28 patients. Brain, 132, 965-981.

Chao, L. L., Haxby, J. V., \& Martin, A. (1999). Attribute-based neural substrates in temporal cortex for perceiving and knowing about objects. Nature Neuroscience, 2, 913-919.

Chouinard, P. A., \& Goodale, M. A. (2010). Category-specific neural processing for naming pictures of animals and naming pictures of tools: An ALE meta-analysis. Neuropsychologia, 48, 409-418.

Connell, L. (2007). Representing object colour in language comprehension. Cognition, 102, 476-485.

Daniel A. W. (2010). Embodied cognition and linguistic comprehension. Studies in History and Philosophy of Science, 41, 294-304.

De Vega, M. (2008). Levels of embodied meaning. From pointing to counterfactuals. In AM. Glenberg, M. de Vega, \& AC. Graesser (eds.), Symbol, Embodiment, and Meaning. Oxford: Oxford University Press.

Eskenazi, T., Grosjean, M., Humphreys, G. W., \& Knoblich, G. (2009). The role of motor simulation in action perception: A neuropsychological case study. Psychological Research, 73, 477-485.

Engelen, J. A. A., Bouwmeester, S., de Bruin, A. B. H., \& Zwaan, R. A. (2011). Perceptual simulation in developing language comprehension. Journal of Experimental Child Psychology, 110 (4), 659 675.

Fodor, J. (1975). The language of thought. Cambridge, MA: Harvard University Press.

Gainotti, G. (2006). Anatomical, functional, and cognitive determinants of semantic memory disorders. Neuroscience and Biobehavioral Reviews, 30, 577-594.

Gallese, V. (2008). Mirror neurons and the social nature of language: The neural exploitation hypothesis. Social Neuroscience, 3, 317-333.

Glenberg, A., \& Robertson, D. (2000). Symbol Grounding and Meaning: A Comparison of HighDimensional and Embodied Theories of Meaning. Journal of Memory and Language, 43, 379401.

Glenberg, A. M., \& Kaschak, M. P. (2002). Grounding language in action. Psychonomic Bulletin \& Review, 9, 588-565.

Gibbs, R. W. Jr. (2006). Embodiment and cognitive science. Cambridge University Press.

Gravetter, F., \& Wallnau, L. (2006). Statistics for the Behavioral Science (7 ${ }^{\text {th }}$ ed.). Wadsworth Publishing.

Hoffman, P., \& Ralph, L. M. A. (2013). Shapes, scents and sounds: Quantifying the full multi-sensory basis of conceptual knowledge. Neuropsychologia, 51, 14-25.

Hauk, O., Johnsrude, I., \& Pulvermuller, F. (2004). Somatotopic representation of action words in human motor and premotor cortex. Neuron, 41(2), 301-307. 
INTERNATIONAL JOURNAL OF ACADEMIC RESEARCH IN PSYCHOLOGY

Vol. 4, No. 1, 2017, E-ISSN: 2312-1882 ๔ 2017 KWP

Jackendoff, R. (2002). Foundation of language. Oxford: Oxford University Press. language processing. Cognitive Science, 30, 733-744.

Kemmerer, D. (2015). Cognitive Neuroscience of Language. New York: Psychology Press.

Kosslyn, S., Ganis, G., \& Thompson, W. (2001). Neural foundations of imagery. Nature Reviews, Neuroscience, 2, 635-642.

Kintsch, W. (1998). Comprehension: A parading for cognition. Cambridge, England: Cambridge University Press.

Levelt, W. J. M. (1989). Speaking: From intention to articulation. Cambridge, MA: MIT Press.

Lallee, S., Madden, C., Hoen, M., \& Dominey, P. F. (2010). Linking language with embodied and teleological representations of actions for humanoid cognition. Frontiers in Neurorobotics, $4(8), 1-12$.

Landauer, T. K., \& Dumais, S. T. (1997). A solution to Plato's problem: The latent semantic analysis theory of acquisition, induction, and representations of knowledge. Psychological Review, 104, 211-240.

Louwerse, M. M., \& Ventura, M. (2005). How children learn the meaning of words and how LSA does it. Journal of Learning Sciences, 14, 301-309.

Louwerse, M. M., \& Connell, L. (2011). A taste of words: Linguistic context and perceptual simulation predict the modality of words. Cognitive Science, 35, 381-398.

Louwerse, M. M. (2011). Symbol interdependency in symbolic and embodied cognition. Topics in Cognitive Science, 3, 273-302.

Martin, A. (2007) the representation of object concepts in the brain. Annual Review of Psychology, $58,25-45$.

Miller, G. A., \& Johnson-Laird, P. N. (1976). Language and perception. Cambridge, MA: Harvard University Press.

Meteyard, L., Cuadrado, S. R., Bahrami, B., \& Vigliocco, G. (2012). Coming of age: A review of embodiment and the neuroscience of semantics. Cortex, 48, 788-271.

Newell, A., \& Simon, H. (1976). Computer science as empirical enquiry: Symbols and search. Communications of the ACM, 19, 113-126.

Oleksandr, V. H., Jean-Christophe, G., Maria, C., \& Grzegorz, P. (2014) From demonstration to theory in embodied language comprehension: A review. Cognitive Systems Research, 29-30, 66-85.

Pecher, D., \& Zwaan, R. A. (2005). Introduction to grounding cognition. Grounding cognition: the role of perception and action in memory, language, and thinking. Cambridge: Cambridge University Press.

Pecher, D., Zeelenberg, R., \& Barsalou, L. W. (2003). Verifying conceptual properties in different modalities produces switching costs. Psychological Science, 14, 119-124.

Pulvermuller, F. (2008). Grounding language in the brain. In M. De Vega, A. Graesser, \& A. M. Glenberg (Eds.) Symbols, embodiment, and meaning (pp. 85-116). Oxford: Oxford University Press.

Pezzulo, G., Barsalou, L. W., Cangelosi, A., Fischer, M. A., McRae, K., \& Spivey, M. (2011). The mechanics of embodiment: A dialogue on embodiment and computational modeling. Frontiers in Cognition, 2(5), 1-21.

Pezzulo, G., \& Calvi, G. (2011). Computational explorations of perceptual symbol systems theory. New Ideas in Psychology, 29, 275-297.

Pylyshyn, Z. W. (1984). Computation and cognition: Toward a foundation for cognitive science. 
INTERNATIONAL JOURNAL OF ACADEMIC RESEARCH IN PSYCHOLOGY

Vol. 4, No. 1, 2017, E-ISSN: 2312-1882 @ 2017 KWP

Cambridge, MA: Bradford Books.

Pulvermuller, F., Haerle, M., \& Hummel, F. (2001). Walking or Talking? Behavioral and Neurophysiological Correlates of Action Verb Processing. Brain and Language, 78, 143-168.

Schank, R. C. (1972). Conceptual dependency: A theory of natural language understanding. Cognitive Psychology, 3, 552-631.

Standfield, R. A., \& Zwaan, R. A. (2001). The effect of implied orientation derived from verbal context on picture recognition. Psychological Science, 12, 153-156.

Vinson, D. P., \& Vigliocco, G. (2008). Semantic feature production norms for a large set of objects and events. Behavior Research Methods, 40, 183-190.

Wilson, M. (2002). Six view of embodied cognition. Psychonomic Bulletin \& Review, 9, 625-636.

Zeki, S. (1990). A century of achromatopsia, Brain, 113, 1721-1777.

Zeki, S. (1997). Cerebral akinetopsia (visual motion blindness): A review. Brain, 114, 811-824.

Zwaan, R. A. (2004). The immersed experiencer: toward an embodied theory of language comprehension. The Psychology of Learning and Motivation (Vol. 44, pp. 35-62). New York: Academic Press.

Zwaan, R. A., Standfield, R. A., \& Yaxley, R. H. (2002). Language comprehends routinely represent the shape of objects? Psychological Science, 13, 168-171.

Zwaan, R. A., Taylor, L. J. Jr. (2006). Seeing, acting, understanding: motor resonance in language comprehension. Journal of Experimental Psychology-General, 135, 1-11. 